\title{
Spatio-temporal prediction of urban expansion using bivariate statistical models: assessment of the efficacy of evidential belief functions and frequency ratio models
}

\begin{abstract}
The urban development process is a continuous and dynamic spatio-temporal phenomenon associated with economic developments and growing populations. Understanding urban expansion processes require models capable of simulating, monitoring, and predicting both urban growth and urban sprawl. In this research, probability-based Evidential Belief Functions (EBF) and Frequency Ratio (FR) models were employed to simulate and to predict the urban expansion probability map of the metropolitan area in Tripoli, Libya. These methods have not been used before in the urban development simulations of cities. By using the geographic information system (GIS), three satellite imageries obtained from 1996, 2002, and 2010 were employed to extract seven urban-deriving factors for the study area. The urban factors are slope, distance to active economic center, distance to central business district (CBD), distance to roads, distance to built-up areas, distance to educational area, and distance to coastal areas. For model calibration, both the EBF and FR models were applied to simulate urban expansion from 1996 to 2002. Data from 2002 to 2010 were used for models validation. Consequently, future suitability maps of urban growth were produced. The validation results indicated $83 \%$ prediction accuracy for the EBF model and $84 \%$ for the FR model. The outcomes established that the models could be employed in the urban expansion modeling of metropolises. The applied models, however, have dynamic and temporal limitations that should be considered in urban growth analysis.
\end{abstract}

Keyword: Urban growth; Dempster-Shafer; Frequency ratio; GIS; Remote sensing; Tripoli 